http://doi.org/10.15359/ree.14-1.2

\title{
Una retrospección y visión de futuro sobre el uso e implementación de las tecnologías de la información y la comunicación, para el aprendizaje virtual en el contexto de la División de Educología de la Universidad Nacional de Costa Rica
}

\author{
Gaby Ulate Solís ${ }^{1}$ \\ Centro de Investigación y Docencia en Educación (CIDE), Universidad Nacional \\ Heredia, Costa Rica \\ Enrique Vílchez Quesada \\ Escuela de Informática, Universidad Nacional \\ Heredia, Costa Rica
}

Recibido 20 de octubre de 2009 • Aceptado 02 de diciembre de 2009

\begin{abstract}
Resumen. La División de Educología de la Universidad Nacional de Costa Rica (UNA) ha sido consciente de la necesaria planificación y de un pensamiento de futuro que demanda una reflexión continua entre lo posible y lo deseable. En este sentido, la formación de los futuros educadores y educadoras del país en sus distintas especialidades, debe fomentar no solamente el uso de la tecnología, sino, también, el desarrollo de un juicio crítico y objetivo que abarque tanto sus ventajas como sus limitaciones en el noble ejercicio de la enseñanza y del aprendizaje. A la luz de estos preceptos, en 2007, emergió la actividad de investigación en docencia denominada Recursos didácticos para el aprendizaje con un enfoque bimodal. Este artículo expone las ideas principales que se han madurado después de una experiencia de más de dos años con este curso, hacia la búsqueda de un redireccionamiento que retoma de manera responsable la planificación, la implementación y las políticas de uso de las tecnologías de la información y la comunicación (TICs) para el aprendizaje bimodal, en el contexto de la División de Educología de la UNA.
\end{abstract}

Palabras clave. Enseñanza, aprendizaje, tecnologías, recursos, bimodal.

\footnotetext{
${ }^{1}$ Profesora de la División de Educología de la Universidad Nacional de Costa Rica, con amplia experiencia docente en el área educativa, en general, y en la enseñanza de las artes y la comunicación visual, específicamente. En la actualidad, colabora con la División de Educología en la actividad de investigación en docencia denominada: "Propuesta del curso Recursos didácticos para el aprendizaje bajo un enfoque bimodal". Correo electrónico: gabyulate@una.ac.cr

${ }^{2}$ Profesor de la Escuela de Informática de la Universidad Nacional de Costa Rica, con experiencia docente en campos diversos, tales como: la matemática y sus aplicaciones, tecnologías de información y comunicación y aprendizaje virtual. Correo electrónico: evilchez@una.ac.cr
}

La Revista Electrónic@ Educare está indizada en el Catálogo LATINDEX.

Artículo protegido por la licencia Creative Commons. 
Abstract. Division de Educología (School of Education for Secondary Level) of Universidad Nacional in Costa Rica, (UNA) has been aware of the necessary planning and thought of a future that demands a continuous reflection between what is possible and what is desirable. In this sense, the formation of future educators of the country in different specialties, should promote not only the use of the technology, but also the development of critical and objective thinking that will embrace its advantages and its limitations in the noble exercise of what is teaching and learning. Under the light of these precepts, in the year 2007, the educational research activity emerged by the name of Didactic Resources for Learning with a bimodal focus. The present article exposes the main ideas that have been matured after an experience of more than two years with this course, and it is also searching for recapturing a responsible way of planning, implementing and managing the policies of ICTs (Information and Communication Technologies) for the bimodal learning in the context of the Division de Educología (School of Education for Secondary Level) of UNA.

Key words. Teaching, learning, technologies, resources, bimodal.

\section{Introducción}

En el contexto de la UNA emergió, en 2006, la iniciativa de incorporar el uso de las tecnologías relacionadas con el aprendizaje virtual a distintos cursos ofrecidos por la División de Educología. Esta División es una unidad académica conformada por diversos profesionales que imparten los cursos de pedagogía a estudiantes de todas las carreras relacionadas con la enseñanza de distintas especialidades, como por ejemplo: Enseñanza de la Matemática, Enseñanza de las Ciencias, Enseñanza de los Estudios Sociales, Enseñanza de las Artes, entre otras (en total son catorce especialidades distintas). Educología pertenece al Centro de Investigación y Docencia en Educación (CIDE) de la UNA.

La División de Educología, ante los retos impuestos por el uso de las tecnologías y sus bondades y limitaciones en el ámbito educativo, creó un proyecto de investigación denominado Docencia virtual. En un inicio, dentro de un marco institucional que desde 2004 ha impulsado una política de desarrollo en la que las tecnologías ocupan un lugar preponderante en la pedagogía, el proyecto se centró en la capacitación de los docentes hacia el uso tecnológico de ciertas herramientas de carácter ofimático, lo que relegó la formación en la conceptualización y diseño de cursos en entornos de aprendizaje virtual, como, originalmente, se había propuesto.

En 2007, en el marco de este proyecto de investigación, surgió otro denominado Propuesta del curso DEX 325 Recursos didácticos para el aprendizaje bajo un enfoque bimodal, en las carreras de Enseñanza de la Matemática y Enseñanza de las Artes y Comunicación Visual. Esta iniciativa contempló, en un período de un año, las etapas de planificación general, operativa, ejecución y evaluación del curso Recursos didácticos para el aprendizaje, con una modalidad que combinó la presencialidad con la virtualidad.

Lo anterior resultó toda una innovación pedagógica dentro de la División de Educología por dos razones principales: en primer lugar, porque docentes y estudiantes, en su mayoría, no tenían formación académica y experiencias previas en el aprendizaje virtual como otra modalidad de estudio y, en segundo lugar, porque en la División de Educología, desde hace varios años, ya se hacía necesario un curso que capacitara y formara a los estudiantes en el uso de ciertas tecnologías, como recursos didácticos dentro de sus distintas especialidades, además del uso de una plataforma de teleformación que, en sí misma, se constituyó en un medio para enseñar recursos didácticos. Un gran reto, si se piensa en las características de una tradición pedagógica dentro de una universidad con una fuerte tendencia hacia lo presencial, y el escaso uso de tecnologías para la enseñanza. Posiblemente, en otros contextos, el proyecto no resultaría tan innovador como ha ocurrido en la UNA. 


\section{Construyendo futuro}

Es importante reconocer que los esfuerzos realizados en la División de Educología de la UNA, parten de un pensamiento docente colectivo que mira hacia el futuro, que pretende construir para el mañana, y que reconoce su responsabilidad como un grupo encargado de formar y de sensibilizar a las nuevas generaciones interesadas en el ámbito educativo de nuestro país.

Pese a ello, las etapas de un proceso de planificación serio pecan por algunos de los inconvenientes suscitados en las transformaciones organizacionales que apuntan al cambio, muchas veces por el poco tiempo para analizar sus limitaciones y posibilidades reales.

Los procesos de diagnóstico realizados en la División de Educología han sido realizados de manera incompleta o superficial, y no se han previsto las necesidades de infraestructura y de equipos informáticos actualizados, al servicio del cuerpo docente y de las grandes cantidades de estudiantes que provienen de zonas rurales del país y que no cuentan con un computador en sus residencias temporales. Esto, desde luego, está ocasionando graves problemas de acceso a los alumnos y a los docentes. El alumnado se queja, constantemente, de sus limitaciones económicas $\mathrm{y}$ de las pocas facilidades para realizar sus tareas virtuales; y muchos profesores se disputan, semana a semana, el uso de los recursos disponibles, por ejemplo, el Vídeo Bin o el único laboratorio de informática con el que cuenta la unidad, para atender más de cuarenta cursos semestre a semestre.

La prospectiva entendida como una anticipación para construir el futuro de manera planificada desde el presente (Prieto, 2008c), en la División de Educología, tiene un comportamiento disgregado, algunos docentes cercanos a las políticas de la unidad apoyan todos los procesos de innovación pedagógica por medio del uso de las tecnologías, y otros se resisten a su uso. Este punto no preocupa tanto, pues en el ámbito educativo el convencimiento profesional es una tarea bipartita entre el tiempo y la capacitación docente.

La gestión académica de la unidad está derivando hacia una planificación general, cuyos resultados convergen en la articulación de distintos proyectos de investigación, incluidos en áreas estratégicas de intereses macros (de la UNA) y micros (de la División, propiamente). La evaluación de todos estos procesos se ha realizado de forma desintegrada, a futuro inmediato es un aspecto fundamental por considerar dentro de la gestión general de la División de Educología.

\section{Sobre el curso Recursos didácticos para el aprendizaje}

En 2007, se inició el reto de incorporar dentro de la formación de futuros docentes, un curso en el que los alumnos lograran comprender y aplicar el uso apropiado de distintos tipos de tecnologías, tanto analógicas como digitales, en y para la docencia.

Lo interesante de esta experiencia ha consistido en el ambiente institucional en el que la idea emergió, en una Universidad que por décadas ha tenido una modalidad presencial y la docencia universitaria ha demostrado un claro énfasis en la experticia por los contenidos, y no tanto en las prácticas pedagógicas de sus profesores. Este último aspecto ha llamado la atención de las autoridades universitarias, pues se ha propuesto, de manera formal, en su gestión, un modelo pedagógico definido y reconocido organizacionalmente.

Al respecto, el curso Recursos didácticos para el aprendizaje, con un enfoque bimodal, se propuso con el objetivo de iniciar el desarrollo de las competencias requeridas por los estudiantes, en la aventura del uso experimental de recursos didácticos basados en tecnologías analógicas, tales 
como la radio, la televisión y el retroproyector y tecnologías digitales, tales como Internet y el uso de software (CmapTools, Photoshop y Dreamweaver), todo ello, con fines educativos.

El plan se inició en 2007, específicamente, en dos carreras: Enseñanza de la Matemática y Enseñanza de las Artes y Comunicación Visual y, en la actualidad, se ha expandido a las catorce carreras a las cuales se dirige. En 2008, los resultados no fueron tan favorables como los obtenidos el año anterior, y las razones se circunscriben a problemas clasificados en las dimensiones pedagógica, tecnológica, de tradición institucional y de las características del sistema educativo nacional. Estas dimensiones fueron identificadas gracias a la aplicación de un instrumento a cuarenta y cinco estudiantes de las carreras Enseñanza de la Matemática, Enseñanza de las Artes y Comunicación Visual, Enseñanza del Inglés y Enseñanza del Español en 2008, además de otro instrumento dirigido a cada uno de los docentes responsables de los grupos.

\section{Problemas por resolver}

Justamente, en el contexto expuesto, ha surgido un problema pragmático muy claro: continuar el curso Recursos didácticos para el aprendizaje con un enfoque bimodal, o volver a impartir el curso con sus características netamente presenciales, sin haber dado cabida a generalizar esta experiencia de manera sistemática a otros cursos que se imparten en la División de Educología.

El problema trasluce elementos de mayor complejidad, pues como se demostró en 2007 (Vílchez y Ulate, 2008), el curso fortalece las competencias de los alumnos en cuanto al uso de recursos didácticos tradicionales (analógicos) e innovadores (digitales), además de mejorar sus condicionamientos cognitivos generando oportunidades para favorecer el autoaprendizaje y el coaprendizaje; sin embargo, ¿por qué en 2008 los resultados no fueron los esperados? Tenemos algunas hipótesis que nos han permitido estructurar una visión de futuro sobre el uso y la implementación de las TICs para el aprendizaje virtual en la unidad académica y que expondremos en este trabajo.

Consideramos que el curso Recursos didácticos para el aprendizaje, con un enfoque bimodal, ofrece, los espacios de enseñanza y de aprendizaje descritos (y, así, podría ocurrir con otros cursos), pero, para ello, el perfil docente, la propuesta pedagógica, los insumos tecnológicos ofrecidos por la institución a profesores y estudiantes, la normativa que rige la implementación de estas modalidades, la resistencia al cambio y la herencia cultural de un sistema educativo, predominantemente, presencial y positivista, requieren de un análisis de construcción de futuro que albergue los retos y las alternativas de solución enmarcados no sólo en la coordinación del curso, sino, también, en la unidad académica en su conjunto, y en la Universidad en general. Hacer explícitos estos aspectos es el principal objetivo de este trabajo.

\section{Justificación del problema}

Construir futuro en la División de Educología no significa ir en concordancia ciega con las nuevas, o relativamente nuevas, tendencias hacia el uso de las tecnologías digitales, significa pensar y repensar la manera en cómo ellas pueden impactar en el futuro de la educación costarricense, en general.

Buscamos soluciones en el mediano y en el largo plazos, que tengan una repercusión en las prácticas educativas de los docentes y, por tanto, en las prácticas de aprendizaje de los estudiantes. Con frecuencia, llamamos a nuestros actuales educandos nativos digitales, pero ¿qué significa, realmente, ser un nativo digital?, ¿bastará con haber crecido presionando botones y conviviendo con pantallas?, 
pareciera que ésta es la convicción irracional de muchos. En realidad, en esta época confusa nos encontramos con salvajes digitales e ilustrados digitales, ¿qué estamos formando?, ¿somos conscientes, en las universidades, de lo que queremos y la dirección contextual que deben llevar las tecnologías hacia la estructuración de una sociedad conformada por personas competitivas, críticas y altruistas.

Creemos que los pasos de transformación de esta utopía a un escenario real, inicia con acciones concretas: aventureras, pero no descabelladas; ambiciosas, pero no descontextualizadas; en el marco de lo idealizable, pero no de lo desproporcionado. A esto, precisamente, aspira responder el proyecto del curso Recursos didácticos para el aprendizaje con un enfoque bimodal. Hay razones muy puntuales ya mencionadas en el apartado anterior, para justificar la continuidad de este trabajo dada su importancia y los aportes institucionales. Sin embargo, ante las dificultades y las limitaciones presentadas en el año 2008, y la necesidad de no desperdiciar los recursos económicos y humanos de la UNA, es fundamental hacer un alto en el camino, no para negar la responsabilidad ya adquirida, sino para realizar un replanteamiento de esta cátedra y, en general, de las políticas de uso e implementación de TICs en la División de Educología de la UNA, las cuales apoyan los procesos de enseñanza y de aprendizaje bimodales. Nos encontramos ante un océano de oportunidades, las que, con el tiempo, creemos, convergerán evolutivamente en una oferta de servicios, en temas relacionados con la calidad de la docencia semipresencial en Costa Rica.

\section{Contexto del problema}

La UNA constituye una de las cuatro universidades públicas de Costa Rica, con una oferta educativa basada en la presencialidad desde su fundación. En la actualidad, el modelo pedagógico en el que se sustenta se fundamenta en los siguientes principios:

- Respeto a la diversidad en todas sus expresiones.

- Respeto y compromiso con la igualdad de oportunidades y con la construcción de una sociedad más justa y equitativa.

- Formación de profesionales solidarios y comprometidos con el bienestar social.

- Flexibilidad para conceptuar el aprendizaje como proceso sociocultural, histórico, dinámico y transformable, posible y que puede construirse de muchas maneras.

- Interacción en los procesos formativos, en los que los conocimientos sean discutidos y enriquecidos permanentemente.

- Formación de un espíritu investigador en los futuros profesionales.

- Creatividad que permita la innovación, así como la utilización de medios, estrategias y recursos de enseñanza en los procesos de mediación pedagógica.

- Disposición para determinar los principios lógicos subyacentes en cada disciplina, que permitan una formación profesional de calidad.

- Evaluación como proceso integral, concertado, permanente, contextualizado y propositivo.

- Mejoramiento continuo en la formación integral de los estudiantes y los procesos de gestión académica-administrativa y paraacadémica.

- Visión prospectiva que permite la planificación estratégica, para el logro de objetivos a mediano y a largo plazo. (Universidad Nacional, 2008, s. p.)

Del modelo pedagógico institucional es rescatable, principalmente, el compromiso organizacional que asumen todos los docentes hacia una cultura de planificación prospectiva, que 
permita, a partir de la adaptación de sus estrategias de enseñanza y de aprendizaje, alcanzar logros que, a mediano y a largo plazo, posibiliten la innovación, el enriquecimiento de los contenidos y la utilización de medios y recursos creativos en la mediación pedagógica. La institución reclama cambios, invita a modernizar, de manera contextual, las prácticas educativas cotidianas, tanto en el ámbito presencial como en el semipresencial.

En particular, la División de Educología, en consistencia con el modelo pedagógico anterior, se ha propuesto los siguientes objetivos:

- Contribuir a la transformación de la sociedad costarricense hacia un desarrollo integral, autónomo y sostenible, mediante la formación de profesionales críticos, independientes, participativos y creativos, con un dominio sólido del conocimiento de su disciplina y su problemática pedagógica.

- Formar, integralmente, educadores para la enseñanza, desde un currículum interdisciplinario que articule la educación con otros campos del conocimiento y fortalezca la identidad profesional de los docentes.

- Crear ambientes académicos favorables a la construcción y reconstrucción del conocimiento, acerca de los procesos de enseñanza y de aprendizaje de una disciplina y de sus vínculos con los procesos educativos en los que ocurren.

- Formar profesionales en educación con sentido de pertenencia cultural, a partir de principios y valores democráticos, que incluyen la tolerancia, la equidad, el respeto por las diferencias, por la vida en todas sus formas, su ambiente y el derecho a la felicidad.

- Desarrollar la vocación investigadora en los educadores y las educadoras, para que fortalezcan los procesos de enseñanza y de aprendizaje, y los vinculen con la realidad educativa en la que trabajan. (Universidad Nacional. División de Educología, 2008)

En el ámbito del uso de los recursos didácticos, la unidad da una especial importancia a la vinculación de las Ciencias de la Educación con otras áreas de conocimiento, específicamente, con el área de las tecnologías analógicas y digitales en cuanto al impacto que producen en el sector educativo, con sus limitaciones y sus fortalezas. Respecto a esto, el curso Recursos didácticos para el aprendizaje representa, con su modalidad semipresencial, una respuesta ante los retos impuestos no solamente por la nueva generación de jóvenes que ingresan a la UNA y a carreras relacionadas con la enseñanza, sino, también, una respuesta en el seno de una institución que, tanto de forma macro como micro, reconoce el valor y la importancia en la capacitación para el uso didáctico de los distintos recursos disponibles de mediación pedagógica, en correspondencia crítica con las nuevas tendencias.

La UNA, además, el 30 de agosto de 2008, aprobó el documento Políticas para la incorporación de las tecnologías de la información y la comunicación en los procesos académicos de la Universidad Nacional, el cual está regulando la incorporación de las tecnologías de información y de comunicación en los procesos académicos institucionales, por medio de una entidad centralizada llamada UNA Virtual, responsable de asesorar, promover y desarrollar iniciativas de incorporación de las TICs en el quehacer académico, además de administrar y desarrollar el Aula Virtual de toda la universidad implementada, de manera oficial, en la plataforma Moodle.

Los resultados no han sido los mejores, pues la centralización de servicios con una alta demanda ha ocasionado una ruptura comunicacional, lentitud en la resolución de problemas, lentitud en la apertura de los cursos y poco codesarrollo de la plataforma Moodle ante las necesidades particulares de la UNA y sus distintas escuelas. 
Por otra parte, vislumbramos a futuro, con cierto temor, pues la parcialización de los procedimientos para la innovación, hace que una unidad académica, al decidir utilizar cierto tipo de TICs en sus procesos educativos, debe hacer la consulta al departamento de UNA Virtual, el cual en muchos casos, se encuentra saturado y con poco personal para entrar de lleno a las necesidades particulares; con esto se nota una pérdida, una debilidad que se concreta en la calidad académica del curso.

La disponibilidad de laboratorios y de licenciamiento de software, es otro de los aspectos que preocupan en el ámbito organizacional, ya que existe un grupo denominado UNA Alternativa, compuesto por académicos de distintas Escuelas que están promoviendo el uso de software libre como solución a las problemáticas causadas por el software privativo, y con una fuerte influencia de la tendencia mundial traída por este movimiento a muchos países, como por ejemplo España. Pese a los esfuerzos, estamos en camino y, aún, en una edad temprana. En la División de Educología de la UNA, además, los recursos son pocos, tanto para adquirir los audiovisuales como para el acceso al único laboratorio disponible para todos los cursos que se imparten semestre a semestre, lo cual es un detalle operativo muy importante por las exigencias de acceso que demanda una modalidad híbrida.

Como parte de este ejercicio de diagnóstico, la caracterización de los alumnos que ingresan a las carreras relacionadas con la enseñanza en la Universidad Nacional de Costa Rica, se resume, de manera genérica, en los siguientes puntos:

- Muchos provienen de zonas rurales en las cuales, durante su educación primaria y secundaria, tuvieron poco contacto con el uso de tecnologías digitales; incluso, muchos entran a la universidad sin saber cómo crear siquiera una cuenta de correo electrónico.

- Se muestran, relativamente, neófitos en actividades relacionadas con la lectoescritura y hábitos de investigación.

- Prefieren, en su mayoría, las clases presenciales y magistrales, y presentan una fuerte resistencia a la modalidad virtual.

- Ignoran cómo trabajar de manera colaborativa o cooperativa, aunque están acostumbrados a relacionarse de forma grupal, pero lo que hacen es dividirse las tareas y unificar, regularmente, sus resultados.

Con este panorama, iniciaremos el análisis de un escenario ideal para cualquier curso semipresencial en la División de Educología de la UNA. Desde hace dos años, con la implementación del proyecto Recursos didácticos para el aprendizaje con un enfoque bimodal, iniciamos este esfuerzo, el cual, mediante esta propuesta, extenderemos, por medio de un ejercicio retrospectivo, en el cual se aprecian la experiencia acumulada hasta la fecha y las características institucionales.

\section{Futuro esperado}

El proyecto Recursos didácticos para el aprendizaje con un enfoque bimodal, emergió con la idea de institucionalizar un curso de pregrado dentro de la formación de futuros educadores, en una modalidad blended-learning (educación semipresencial) que lograra aproximar a los estudiantes a otras realidades educativas concordantes con el uso de las tecnologías de la información y la comunicación. Durante toda la experiencia desarrollada en torno al proyecto, hemos consolidado un escenario ideal que debería orientar cualquier iniciativa relacionada con el aprendizaje virtual dentro de la División de Educología de la UNA. Este escenario se circunscribe en los siguientes aspectos: 
- Dimensión pedagógica. Los docentes presentan una formación y una experiencia adecuadas en el diseño y el manejo de un entorno de aprendizaje virtual; además, son conscientes de las diferencias y las similitudes entre los espacios educativos presenciales y virtuales. Se sienten comprometidos con una modalidad educativa bimodal, y reconocen el aporte que ésta puede proporcionar en cuanto al desarrollo de competencias, lo cual, sin el uso de esta modalidad, sería más difícil integrar en la formación de los estudiantes. Los docentes presentan las habilidades necesarias para la elaboración de material didáctico dirigido a este tipo de escenarios educativos. Finalmente, los profesores tienen conocimiento sobre estándares de calidad en la educación a distancia.

- Dimensión tecnológica. Los profesores utilizan la tecnología con una actitud pedagógicamente crítica, pues son usuarios y, al mismo tiempo, codesarrolladores, quienes definen, con claridad, sus necesidades y los aspectos en los cuales la plataforma Moodle de la UNA debería converger, con ayuda del grupo UNA Virtual. Los docentes tienen formación tanto en el uso técnico de las tecnologías analógicas como también de las digitales. Poseen conocimiento de experiencias internacionales sobre el uso de estas tecnologías en el sector educativo. Son usuarios activos de la Red y las tecnologías Web 2.0, y reconocen, desde su visión de consumo, sus ventajas y limitaciones.

- Tradición institucional. La UNA reconoce la importancia y la necesidad de crear espacios educativos semipresenciales y virtuales. Funda un Centro de Materiales Audiovisuales, el cual apoya los procesos de enseñanza y de aprendizaje a distancia. En su normativa institucional, en cuanto al reglamento de evaluación de los aprendizajes y a los derechos y deberes, tanto de los académicos como de los estudiantes, se brinda una atención particular a las modalidades semipresenciales y virtuales. Institucionalmente, se brinda a los distintos grupos interdisciplinarios el tiempo necesario dentro de su jornada, para la conceptualización, el diseño y la implementación de cursos y programas dentro de estas modalidades.

- Sistema educativo nacional. El Ministerio de Educación Pública (MEP), con apoyo de los académicos de la División de Educología de la UNA, inicia procesos de formación y de capacitación docente en tutoría virtual, entornos de aprendizaje virtuales y capacitación específica en didáctica, pedagogía y andrología.

Por otra parte, vislumbrando el escenario posible en cada una de estas dimensiones, pretendemos, en un plazo máximo de cinco años, obtener los siguientes logros:

- Dimensión pedagógica. Docentes capacitados en el área de la tutoría virtual, al menos con un curso aprobado por una organización internacional. Hay claridad sobre el perfil de los profesores que constituyen las distintas cátedras de los cursos que se imparten de forma semipresencial, lo cual proporcionará insumos importantes en la contratación de personal. Se consolidará un grupo de tres o cuatro investigadores en el área, para determinar, en el contexto de la UNA, ventajas, desventajas y necesidades ante las exigencias de una modalidad blended-learning. Se contará con un grupo de tres a cuatro profesionales que, de manera permanente, se dedicarán a elaborar y reelaborar materiales didácticos para el curso Recursos didácticos para el aprendizaje u otros que se impartan de forma bimodal.

- Dimensión tecnológica. Los docentes que formarán parte de las cátedras de cursos semipresenciales se capacitarán en el uso de distintos recursos tecnológicos tanto analógicos como digitales, y recibirán el estímulo de contar con una beca semestral para participar en un curso en el Instituto de Capacitación y Asesoría Informática, o, bien, en el programa 
Identidad Cultural, Arte y Tecnología, ambos centros pertenecen a la UNA. Anualmente, la coordinación general de las cátedras de los cursos bimodales, recogerá información para detectar las debilidades y las fortalezas de la plataforma Moodle implementada en la UNA, esta información realimentará los esfuerzos del departamento UNA Virtual para robustecer y adecuar la plataforma institucional. Además, un grupo de dos o tres especialistas actualizará a todas las cátedras con experiencias internacionales, para que alimenten el quehacer académico en el contexto de todos los cursos semipresenciales.

- Tradición institucional. Se solicitará a la Dirección de la Unidad Académica un pronunciamiento anual, si fuera necesario, de parte del Consejo de Unidad y Consejo de Facultad a la Rectoría para regularizar, en el marco jurídico, los derechos y las obligaciones de académicos y estudiantes en el contexto de una modalidad semipresencial y/o virtual.

- Sistema educativo nacional. La División de Educología ofrecerá prestación de servicios a la comunidad costarricense, en general, para la implementación exitosa de cursos bimodales o virtuales. El proceso iniciará sin ningún costo y ofrecerá asesoría al MEP en temas relacionados con la tutoría virtual y la complejidad de los entornos de aprendizaje virtual.

La propuesta pedagógica macro de los cursos con características virtuales y sus objetivos con la modalidad blended-learning, se presentan en los siguientes apartados.

\section{Propuesta pedagógica}

En los cursos de la División de Educología con un enfoque bimodal, el uso, la construcción y las aplicaciones, en el ámbito educativo, de diversos recursos didácticos, tanto analógicos como digitales, se fundamenta en el reconocimiento del aprendizaje como un producto social de acuerdo con Vygotsky (citado por Espiro, 2008a) y como un proceso activo según Ausubel y Bruner (citados por Espiro), a partir del cual los alumnos construyen o reconstruyen ideas, conceptos, soluciones, estrategias, conjeturas y aplicaciones de la teoría a situaciones reales o hipotéticas, en los diversos contextos educativos que caracterizan al sistema educativo nacional.

Se fomenta la construcción social del conocimiento mediante el trabajo en equipo, cooperativo y colaborativo. Se espera que los alumnos se apropien de habilidades y destrezas de investigación, que les permita tener una mayor independencia sobre sus estructuras de pensamiento, aprendizaje de estrategias de aprendizaje (aprender a aprender) y el desarrollo de un pensamiento crítico por medio del ejercicio dialógico, el respeto por los saberes individuales y colectivos y la creación conjunta de nuevas ideas, tal y como nos lo plantea la educación liberadora propuesta por Paulo Freire (citado por Espiro, 2008b).

El andamiaje citado por Bruner y el aprendizaje significativo desarrollado por Ausubel, en muchas de las estrategias didácticas que caracterizan a los cursos, cobran una posición central tanto en el ejercicio de la tutoría virtual y presencial, como en las actitudes que se espera desarrollar en los educandos. El docente asumirá el reto de elaborar y seleccionar materiales de apoyo (lecturas, presentaciones, links, recursos de la Web 2.0, problemas) que inciten el descubrimiento de técnicas y métodos en los que el alumno encuentre los espacios necesarios para una construcción y aplicación personal y colectiva de los conocimientos relacionados con cada unidad temática.

Finalmente, esta propuesta pedagógica toma en consideración, también, la importancia del aprendizaje receptivo y el condicionamiento operante propuesto por Skinner (citado por Espiro, 
2008a), en el ejercicio de la tutoría virtual estimulando la práctica de una conducta social adecuada y los fundamentos de una comunicación asertiva, respetuosa y tolerante, utilizando las distintas herramientas de comunicación tanto sincrónicas como asincrónicas del aula virtual. Además, se reconoce el valor del aprendizaje memorístico como puente para conciliar los conocimientos teóricos previos, con estrategias de enseñanza no mecanicistas.

\section{Objetivos}

El objetivo general que se ha dispuesto en la visión de futuro sobre el uso y la implementación de las TICs para el aprendizaje virtual en el contexto de la División de Educología de la UNA, es el siguiente: desarrollar procesos formativos en los educandos hacia el uso, la construcción y la aplicación de las distintas unidades temáticas de los cursos, mediante una construcción individual y social del conocimiento que promueva una conciencia pedagógica crítica y constructiva, de las aplicaciones y las limitaciones que tienen las tecnologías analógicas y digitales en el ámbito educativo.

Como objetivos específicos, proponemos los siguientes:

- Identificar ventajas y desventajas que aportan las tecnologías analógicas y digitales en los diversos contextos, de acuerdo con el área cognitiva de cada disciplina y área en estudio.

- Identificar fortalezas y debilidades de uso de los distintos tipos de recursos didácticos que aportan las tecnologías analógicas y digitales al sistema educativo costarricense.

- Utilizar distintos tipos de tecnologías analógicas y digitales como apoyo en los procesos de enseñanza y de aprendizaje.

\section{Resultados esperados}

Los resultados que se espera obtener con las distintas cátedras de los cursos que se circunscriban con un enfoque bimodal, se centran en los agentes principales involucrados con sus procesos de implementación: los estudiantes, los docentes, el departamento de UNA-Virtual y la institución, en general.

Los resultados esperados, en un lapso de tres años, son los siguientes:

- La existencia de un coordinador general de todos los cursos tanto en sus características presenciales como virtuales, un grupo de dos profesionales con una carga de un cuarto de tiempo cada uno, destinada a la investigación de las implicaciones teóricas que emergen en la práctica educativa de los distintos cursos, un experto de contenido y un diseñador gráfico para la elaboración y la reelaboración de materiales didácticos, con una carga académica de, al menos, un cuarto de tiempo.

- Un equipo de cinco docentes con formación académica, debidamente acreditada, en el área de la tutoría virtual. Se espera que estos profesores se encuentren capacitados, al menos, con dos cursos relacionados con la tutoría virtual y el manejo de entornos virtuales de aprendizaje.

- Un profesional del departamento de UNA Virtual integrado, debidamente, con el proyecto y su coordinador general, para el manejo administrativo de la plataforma y la oferta de servicios de capacitación y consultas técnicas, tanto a profesores como a estudiantes, relacionados con el uso pedagógico y técnico de la plataforma Moodle. 
- La publicación de un artículo anual en una revista científica internacional sobre los avances teóricos suscritos a las prácticas educativas en torno a los distintos cursos bimodales, los alumnos, la plataforma de teleformación utilizada y las prácticas docentes presenciales y virtuales más adecuadas, en el contexto de la UNA.

- Una mediación pedagógica de todos los materiales y recursos didácticos utilizados en las diferentes cátedras de los cursos, tanto para el trabajo presencial como virtual.

- La capacitación permanente de los docentes de las cátedras en el uso de distintos tipos de recursos didácticos, tanto analógicos como digitales. Se pretende que cada docente se capacite con, al menos, un curso anual.

- La promoción de acciones para crear una conciencia institucional sobre la necesidad de incorporar y diferenciar, en el marco jurídico de la Universidad, los derechos y las obligaciones de académicos y estudiantes, en el contexto de una modalidad semipresencial y/o virtual.

- Una propuesta de cursos madura y validada en prácticas de aprendizaje, estructura y estrategias de evaluación que permitan migrar la experiencia a otros cursos de la División de Educología y, a futuro, ofrecer servicios de formación al MEP, en esta materia.

\section{Aspectos operativos}

Los aspectos operativos relacionados con las políticas de uso e implementación de TICs para el aprendizaje virtual en la División de Educología de la UNA, se especifican a continuación. La exposición se divide en: prácticas de aprendizaje y tecnologías, materiales didácticos, tutoría prevista, administración del sistema, evaluación y seguimiento.

\section{Prácticas de aprendizaje y tecnologías}

En los cursos de la División de Educología que utilicen un enfoque blended-learning, el trabajo se realizará mediante la acción conjunta de profesor(a) y estudiantes, en ellos se plantean y desarrollan, mediante la investigación y el trabajo cooperativo/colaborativo, los diferentes temas que interesa conocer. El(la) profesor(a) cumple una función de facilitador(a) en la construcción del conocimiento, los estudiantes son los responsables de llevar a buen término su proceso de aprendizaje por medio de las actividades propuestas.

Es esencial el trabajo en equipo, la búsqueda de consenso, la lluvia de ideas para obtener significados, la búsqueda de información, la elaboración de categorías y los procesos de análisis y de síntesis, en la adquisición y la reconstrucción del conocimiento.

\section{Materiales didácticos}

Los materiales de estudio que se utilizarán en los distintos cursos bimodales dentro de la División de Educología, serán seleccionados y elaborados, adaptados y editados por el coordinador general y de cátedra de cada curso, mediante la colaboración de un diseñador gráfico y los docentes-tutores. Los documentos o lecturas tendrán formato PDF, dada su facilidad de acceso y de optimización en el peso de los archivos. 


\section{Tutoría prevista}

El grupo de profesionales que se encargará de acompañar a los alumnos durante las dieciocho semanas de los distintos cursos bimodales, estará conformado por tutores debidamente capacitados en el área, quienes asumirán la responsabilidad de un grupo con, a lo sumo, treinta estudiantes.

Existirá la figura de un tutor general (coordinador), quien tendrá asignadas las siguientes funciones: velar por la calidad de los servicios académicos y administrativos ofrecidos a los estudiantes, proporcionar apoyo a los docentes (sobre todo, a aquellos que se inician y los introducen en el enfoque pedagógico de los cursos), coordinar con la persona encargada en el departamento de UNA Virtual la apertura de cursos y procesos de capacitación y encauzar las tareas de investigación en torno a este proyecto. Además, el coordinador general identificará los puntos débiles en cuanto a contenidos, dominio tecnológico y tutoría virtual de su grupo de colaboradores, y gestionará ante las autoridades de la unidad académica el presupuesto requerido, con el objetivo de capacitar a los docentes en las áreas prioritarias.

\section{Administración del sistema}

En el contexto de la UNA, el departamento de UNA Virtual, oficialmente, tiene a su cargo la administración tecnológica de la plataforma institucional, en este caso y hasta la fecha, la plataforma Moodle. Desde esta perspectiva, compete a este organismo la apertura de los cursos en toda la Universidad; sin embargo, en muchas ocasiones, se ha comprobado, en la práctica, que esto tiene repercusiones importantes en el tiempo de creación espacial de cursos virtuales o semipresenciales. En este sentido, el coordinador general del proyecto velará por mantener una comunicación constante con el representante del departamento UNA Virtual a cargo, tratando de prever inconvenientes que pongan en riesgo el inicio exitoso de los cursos en sus componentes virtuales.

Los materiales de los cursos serán entregados a los estudiantes en un CD durante las dos primeras semanas del período lectivo, las que se dictarán de manera presencial.

El coordinador general se encargará también de gestionar los procesos para evaluar la calidad de los cursos ofrecidos en cuanto a: prácticas de aprendizaje, estrategias de evaluación, actualización de los contenidos, bibliografía y lincografía, calidad de la tutoría, fortalezas y debilidades en la plataforma de teleformación, acceso a los laboratorios institucionales y rendimiento académico de los estudiantes.

\section{Evaluación}

La evaluación de las distintas cátedras en la División de Educología que utilicen un enfoque bimodal, integrará indicadores de calidad distribuidos en los siguientes aspectos: la gestión de los cursos, los materiales didácticos, los medios y tecnologías seleccionadas, la evaluación de los aprendizajes y la infraestructura que dará soporte a la implementación. Los indicadores han sido tomados como puntos de referencia del documento Informe final de la CONEAU (Becerra, Bavio, Díaz, Maggio, Martínez, Mena et al., s. f.), y han sido adaptados a las características del proyecto que aquí nos ocupa, en el marco de acción de la División de Educología de la UNA. Las líneas siguientes explican el contexto particular en el cual será imprescindible efectuar los procesos de autoevaluación en las dimensiones anteriormente establecidas. 


\section{Gestión de los cursos}

Los cursos con un enfoque blended-learning, como en algún momento ya fue explicado, contarán con un coordinador general. Con el objetivo de garantizar el buen funcionamiento de su gestión académica y administrativa, será indispensable que la persona más próxima, de acuerdo con la jerarquía organizacional, realice una evaluación sobre el cumplimiento de sus principales funciones, las cuales pueden resumirse en:

- Cuidar la calidad de los servicios académicos y administrativos ofrecidos a los estudiantes.

- Brindar apoyo a los docentes en asuntos tanto académicos como administrativos.

- Realizar procesos de inducción para aquellos profesores que se inician con el enfoque pedagógico de los cursos.

- Coordinar con la persona encargada en el departamento de UNA Virtual la apertura de cursos y procesos de capacitación.

- Analizar las fortalezas y las debilidades de la plataforma institucional.

- Liderar los procesos de investigación en torno al proyecto.

- Liderar los procesos de mejoramiento de las cátedras en cuanto a contenido, prácticas de aprendizaje, experiencias de evaluación, dominio tecnológico y tutoría virtual.

- Gestionar ante las autoridades de la unidad académica el presupuesto requerido, con el objetivo de capacitar a los docentes en las áreas prioritarias.

- La dirección de la División de Educología será la responsable de velar por el cumplimiento de estas tareas, en la figura del coordinador general.

\section{Materiales didácticos}

Los materiales didácticos seleccionados y elaborados para las cátedras de los cursos serán el resultado de un proceso continuo y colaborativo, por parte de los docentes/tutores quienes tendrán la responsabilidad de elaborar y/o adaptar estos recursos a sus distintas especialidades y/o áreas. En conjunto, se diseñarán guías didácticas que integrarán todos los materiales por área tanto generales como específicos de las distintas disciplinas; estas guías servirán como hilo conductor de contenido y experiencias de aprendizaje para futuras promociones de los cursos. Con todo ello, el proceso de evaluación de estos materiales, a la luz de la percepción de los estudiantes, deberá contemplar los siguientes aspectos:

- Que sean apropiados para la educación a distancia.

- Que sean claros y tengan una relación directa con los objetivos de aprendizaje de los cursos.

- Que hagan referencia a aplicaciones concretas de los temas de los cursos a la disciplina en estudio.

- Que tengan una presentación que incita a su lectura.

- Que ofrezcan información completa, pero no acabada, y que muestre al lector otras fuentes de información a materiales complementarios.

Los docentes que conforman las distintas cátedras tendrán un lugar preponderante en los procesos de autoevaluación dirigidos a mejorar la calidad de los materiales que caracterizan a los cursos, por tanto, el coordinador general, al finalizar cada período lectivo, asumirá la responsabilidad 
de convocar a todos sus colaboradores para analizar los resultados obtenidos en las evaluaciones de la percepción estudiantil, e iniciar las acciones que, en conjunto, conducirán a una reelaboración y/o reselección de los materiales.

\section{Medios y tecnologías}

En las cátedras de los cursos bimodales de la División de Educología de la UNA, los docentes asumirán la responsabilidad de analizar, en conjunto con el coordinador general, el impacto que tienen, en los procesos de enseñanza y de aprendizaje, las prácticas de aprendizaje implementadas en cada período lectivo.

Este aspecto será esencial para decidir cuáles tecnologías constituirán el soporte en la mediación pedagógica de las experiencias que conducirán a los educandos hacia el aprendizaje tanto individual como cooperativo. En este contexto, la observación de los registros dejados por los estudiantes en el aula virtual (en sus distintas asignaciones), se convertirá en el insumo de información básica para respaldar o refutar las decisiones tomadas por el grupo docente hacia el uso de otras tecnologías y medios que mejoren los resultados esperados en cuanto al desarrollo de habilidades metacognitivas y de trabajo colaborativo.

Además, el coordinador general del curso se encargará, también, de valorar el desempeño de los docentes en cuanto a las habilidades tecnológicas demostradas durante y al finalizar el período lectivo, encauzando las fuerzas administrativas necesarias para facilitar espacios de capacitación en primera instancia, por medio de la colaboración del departamento de UNA Virtual y, a más largo plazo, mediante la inscripción en cursos especializados.

Finalmente, se aplicará un instrumento de evaluación a los estudiantes y docentes participantes de los cursos en sus distintas áreas y especialidades, para valorar las ventajas y las limitaciones de la plataforma Moodle con sus características de instalación e implementación institucionales. La información suministrada y analizada por el coordinador general brindará información precisa al departamento de UNA Virtual sobre las necesidades y fortalezas de la plataforma, con el objetivo de realimentar su trabajo hacia la búsqueda de una herramienta tecnológica que responda a las demandas sentidas por la comunidad universitaria.

\section{Evaluación de los aprendizajes}

Éste es uno de los aspectos más importantes en el contexto del proyecto aquí expuesto. Creemos como ya se ha manifestado, que los cursos basados en un enfoque bimodal pueden potenciar el desarrollo de habilidades y destrezas fundamentales para un educador del siglo XXI. Estas habilidades y destrezas deseables en los estudiantes, responden a una serie de estrategias materializadas en las prácticas de aprendizaje y, sobre todo, explicitadas en la forma en cómo, a la luz de este mapa de prácticas, se evalúa el progreso alcanzado por los alumnos y su seguimiento. La evaluación de los aprendizajes debe valorarse tomando en consideración los siguientes puntos:

- La evaluación de los aprendizajes en los cursos facilita la determinación del nivel de progreso alcanzado por los educandos en cuanto a: contenido, habilidades de auto aprendizaje y habilidades para el trabajo colaborativo.

- La evaluación es consistente con las prácticas de aprendizaje. 
- La evaluación es consistente con un enfoque alternativo, brinda espacios para realimentar a los educandos sobre sus avances, logros y debilidades; está enfocada a un mejoramiento continuo y no a un resultado absoluto.

- La evaluación contempla aspectos tanto cualitativos como cuantitativos.

- Los estudiantes conocen, de manera precisa, los aspectos en los cuáles serán evaluados en todas las actividades realizadas en los cursos.

El coordinador general de los cursos se encargará de colaborar con su grupo de docentes para ir transformando, poco a poco, la fuerte tendencia institucional en el contexto de la UNA, en donde se ha mantenido, durante años, una clara tradición positivista.

\section{Infraestructura institucional}

La infraestructura y la compra de equipo audiovisual constituyen, para este proyecto, un reto muy importante. La UNA requiere crear espacios académicos apropiados para los estudiantes que cursan materias en una modalidad bimodal o virtual. Los esfuerzos realizados en el ámbito institucional aún son insuficientes para cubrir con una demanda que se ha delegado al alumno, y se le ha obligado a invertir recursos por cuenta propia, para garantizar su acceso a las tareas virtuales asignadas en estos programas.

La institución tiene a su cargo la responsabilidad de tomar parte en este aspecto. Se espera que los informes de autoevaluación arrojados a la luz de este proceso, sirvan para crear la presión institucional necesaria, que en un mediano y en un largo plazos hagan tomar conciencia a las autoridades universitarias sobre esta problemática. Desde esta perspectiva, la evaluación de la infraestructura institucional se fundamentará en:

- Identificar las carencias, en cuanto al uso de laboratorios, de acuerdo con la percepción docente y estudiantil.

- Identificar las carencias en la División de Educología de la UNA, en relación con el equipo audiovisual y computacional disponible.

- Identificar las principales carencias de infraestructura y de equipo que representan los mayores obstáculos para crear condiciones de calidad en este proyecto.

Muchos de los aspectos anteriormente citados ya son conocidos dentro de la cultura institucional y en la práctica educativa de los docentes; sin embargo, nos parece esencial sistematizarlo mediante la aplicación de instrumentos que recojan la percepción de los agentes involucrados.

\section{Seguimiento}

El seguimiento del proyecto será ejecutado por el coordinador general en colaboración con todos los profesores de las distintas cátedras de los cursos bimodales.

En este proyecto, el seguimiento se concibe como un aspecto esencial para:

- Construir, seleccionar y/o reelaborar nuevos materiales didácticos.

- Solucionar, a corto plazo, problemas de contenido, tecnológicos o de relaciones humanas. 
- Garantizar una práctica educativa homogénea en cuanto al enfoque pedagógico implementado por los docentes.

- Proponer innovaciones relacionadas con las prácticas de aprendizaje y, a la luz de estas nuevas propuestas, definir el uso de otras herramientas tecnológicas.

- Diseñar, de manera paulatina, una serie de guías didácticas que constituyan una memoria escrita sobre los materiales básicos en los cuales se fundamentan los cursos, los aportes personales de cada docente en su disciplina y área específica (tanto en experiencias de aprendizaje como de evaluación) y ejemplos de recursos realizados por los mismos estudiantes.

Las estrategias que utilizará el coordinador se sustentarán en: un seminario permanente, un taller de reflexión pedagógica al finalizar cada período lectivo y una bitácora personal del trabajo realizado por cada uno de los docentes mediante un blog.

El seminario permanente será convocado por el coordinador de manera quincenal y, en estos espacios de trabajo, se procederá a:

- Introducir a los nuevos docentes en el enfoque pedagógico del curso.

- Proponer nuevos materiales didácticos de manera conjunta.

- Proponer nuevas prácticas de aprendizaje y el uso de otras tecnologías digitales o analógicas.

- Ofrecer a las y los docentes charlas formativas en aspectos tecnológicos y de tutoría virtual (por parte del departamento UNA Virtual).

- Elaborar, en conjunto y durante cada semestre, guías didácticas digitales con material didáctico y experiencias de aprendizaje probadas por los mismos docentes en su práctica profesional.

- Ofrecer espacios para que los estudiantes de distintas disciplinas puedan compartir con otros profesores sus realizaciones creativas (esto permitirá seleccionar, de forma consensuada, cuáles trabajos podrían ser incluidos en las guías didácticas).

Al finalizar cada semestre, el coordinador general convocará a un taller de reflexión pedagógica con la intención de:

- Identificar fortalezas y debilidades en:

- La propuesta pedagógica de los cursos.

- Las prácticas de aprendizaje y de evaluación.

- Los materiales de estudio ofrecidos a los estudiantes.

- El equipo audiovisual y computacional disponible y sus horarios de uso.

- Identificar las fortalezas y los requerimientos de la plataforma institucional Moodle.

- Definir las necesidades sentidas por el grupo de docentes colaboradores y, a la luz de estos resultados, implementar futuros procesos de capacitación.

- Brindar un informe sobre el trabajo realizado por la coordinación durante el período y los procesos de investigación, encauzados en torno al proyecto.

- Ofrecer la oportunidad para el diálogo abierto sobre el trabajo realizado por todos y todas.

Finalmente, el coordinador general abrirá un espacio privado a cada uno de los docentes de los distintos cursos, quienes tendrán la responsabilidad de moderar, de forma semanal, durante todo el semestre, un blog cuyos únicos invitados serán los colegas de la cátedra. El blog conformará 
una bitácora personal de los profesores sobre sus experiencias semanales con sus estudiantes ante: cada uno de los contenidos, las prácticas de aprendizaje asignadas, las experiencias de evaluación, los materiales utilizados, los materiales desarrollados por los estudiantes, las dificultades presentadas en la tutoría y las necesidades sentidas junto con sus posibles soluciones. El blog permitirá al grupo de profesores comunicar y socializar durante el proceso, aspectos tanto positivos como negativos que serán idealmente realimentados por todos, de manera oportuna. Es reconocible en esta iniciativa, el efecto mariposa que podría ocasionar, pues si el docente, de manera previa, considera el blog como un medio de control, por parte del coordinador general, perderá su sentido didáctico y colaborativo.

\section{Conclusiones}

En este documento, se han propuesto las bases de las cuales dependerá la exitosa transición en cuanto al uso e implementación de las tecnologías de la información y la comunicación para el aprendizaje virtual, en el contexto de la División de Educología de la UNA. Estas bases son el resultado de una experiencia docente y de investigación de varios años, y podrían resultar de interés a otras instituciones que se encuentren en el mismo marco de transición. Las políticas de uso e implementación de TICs expuestas constituyen insumos, ante los cuales otras instituciones u organizaciones podrían tomar importantes decisiones para la actualización de sus currículos, perfiles docentes y manejo administrativo de sus cursos virtuales o bimodales.

\section{Referencias bibliográficas}

Becerra, M., Bavio, E., Díaz, E., Maggio, M., Martínez, L., Mena, M., et al. (s. f.). Comisión asesora de educación a distancia. Informe final. Argentina: Ministerio de Educación, Ciencia y Tecnología.

Espiro, S. (2008a). Aprendizaje. En: Antología utilizada en el Posgrado de especialización en entornos virtuales del aprendizaje. OEI-Virtual Educa.

Espiro, S. (2008b). Aprendizaje adulto. En: Antología utilizada en el Posgrado de especialización en entornos virtuales del aprendizaje. OEI-Virtual Educa.

Prieto, D. (2008c). Planificar para construir futuro. En: Antología utilizada en el Posgrado de especialización en entornos virtuales del aprendizaje. OEI-Virtual Educa.

Vílchez, E. \& Ulate, G. (2008). Curso Recursos didácticos para el aprendizaje. Una experiencia en la virtualidad. Revista Cognición, $N^{o} 14,52-80$.

Universidad Nacional. (2008). Modelo pedagógico institucional. Recuperado el 5 de noviembre de 2008, de http://www.una.ac.cr

Universidad Nacional. División de Educología. (2008). Misión y Visión de la Unidad Académica. Recuperado el 5 noviembre de 2008, de http://www.una.ac.cr/cide/Educologia/index.html 


\section{Otras fuentes consultadas}

Duart, J. \& Sangrà, A. (2000). Aprender en la virtualidad. Barcelona, España: Gedisa.

García, A. (1998). Indicadores para la evaluación de la enseñanza en una universidad a distancia, Revista Iberoamericana de Educación a Distancia, $N^{\circ} 1,63-85$.

Juárez, A. \& Assad, C. (2001). Gestión y evaluación de un sistema a distancia, en Red de Educación a Distancia de las Universidades Nacionales Región Centro-Oeste, Nº1, 14-18.

Prieto, D. (2008a). Evaluación y seguimiento. En: Antología utilizada en el Posgrado de especialización en entornos virtuales del aprendizaje. OEI-Virtual Educa.

Prieto, D. (2008b). Fase de planificación operativa. En: Antología utilizada en el Posgrado de especialización en entornos virtuales del aprendizaje. OEI-Virtual Educa.

Prieto, D. \& Van de Pol, P. (2006). E-learning comunicación y educación. El diálogo continúa en el ciberespacio. San José, Costa Rica: Radio Nederland Training Centre. 